\title{
Review Article \\ Circulating Biomarkers of Interstitial Lung Disease in Systemic Sclerosis
}

\author{
Harpreet K. Lota and Elisabetta A. Renzoni \\ Interstitial Lung Disease Unit, Royal Brompton Hospital, London, SW3 6NP, UK \\ Correspondence should be addressed to Elisabetta A. Renzoni, e.renzoni@rbht.nhs.uk \\ Received 18 May 2012; Revised 23 July 2012; Accepted 28 July 2012 \\ Academic Editor: Luis R. Espinoza
}

Copyright (C) 2012 H. K. Lota and E. A. Renzoni. This is an open access article distributed under the Creative Commons Attribution License, which permits unrestricted use, distribution, and reproduction in any medium, provided the original work is properly cited.

\begin{abstract}
Interstitial lung disease (ILD) is a major cause of morbidity and mortality in patients with systemic sclerosis (SSc). Although a large proportion of SSc patients have only limited interstitial involvement with an indolent course, in a significant minority ILD is progressive, requiring prompt treatment and careful monitoring. One of the main challenges for the clinician treating this highly variable disease is the early identification of patients at risk of progressive ILD, while avoiding potentially toxic treatments in those whose disease is inherently stable. Easily available and repeatable biomarkers that allow estimation of the risk of ILD progression and early response to treatment are highly desirable. In this paper, we review the evidence for circulating biomarkers with potential roles in diagnosis, monitoring of disease activity, or determining prognosis. Peripheral blood biomarkers offer the advantages of being readily obtained, non-invasive, and serially monitored. Several possible candidates have emerged from studies performed so far, including SP-D, KL-6, and CCL18. Presently however, there are few prospective studies evaluating the predictive ability of prospective biomarkers after adjustment for disease severity. Future carefully designed, prospective studies of well characterised patients with ILD, with optimal definition of disease severity and outcome measures are needed.
\end{abstract}

\section{Introduction}

Systemic sclerosis (SSc) is a multisystem, autoimmune connective tissue disease, characterised by excessive extracellular matrix deposition, with remarkable heterogeneity in organ involvement pattern and prognosis. Pulmonary involvement, due to pulmonary fibrosis or pulmonary hypertension, is the leading cause of mortality $[1,2]$. The pathogenesis of pulmonary fibrosis in SSc involves a complex combination of epithelial and endothelial cell injury with inflammatory and immune activation. Occurring in response to unknown initiating factors, the interaction between vascular, epithelial, and immune dysfunction leads to dysregulated fibroblast activation and increased extracellular matrix production [3]. This paper will focus on the circulating biomarkers for SScassociated interstitial lung disease (SSc-ILD), as summarised in Figure 1.

A degree of interstitial involvement is present in the majority of patients with SSc, although severity of lung disease at presentation and subsequent longitudinal behaviour are highly variable. In view of the marked variability in the natural history of SSc-ILD, markers of the likelihood of ILD progression are needed in clinical management. Patients with a recent diagnosis of SSc are more at risk of ILD progression, as the rate of forced vital capacity (FVC) decline is highest during the first five years since diagnosis [4]. The severity of ILD also has clear prognostic implications. Decreased FVC and diffusing capacity of the lung for carbon monoxide (DLCO) have been repeatedly identified as risk factors for progression, as has the extent of ILD on CT [5-7]. Recently, Goh et al. have proposed a simple staging system which subgroups SSc-ILD into limited and extensive, based on rapid estimation of CT extent, integrated, if necessary, by FVC levels. This has been found to be more accurate prognostically than either CT or FVC in isolation [8] and can be easily applied in the clinical setting to provide rapid estimation of likelihood of ILD progression. By contrast, bronchoalveolar lavage (BAL) findings provide only limited prognostic information and are not linked to long-term survival or the rapidity of progression of 


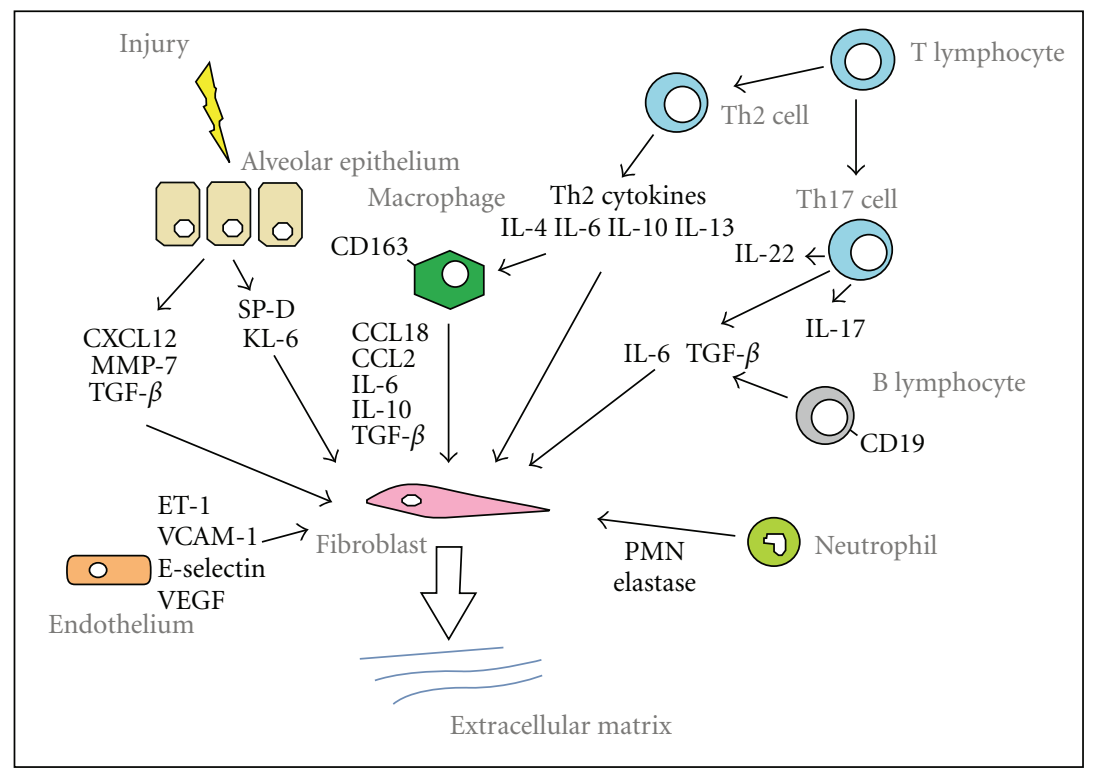

FIgURE 1: Potential biomarkers in SSc-ILD.

lung disease $[9,10]$, although may provide insights into pathogenetic mechanisms. Histological pattern is also not linked to likelihood of disease progression. In contrast to the idiopathic setting, a pattern of usual interstitial pneumonitis (UIP), seen only in a minority of patients, is not associated with a significantly worse survival than the nonspecific pneumonitis (NSIP) pattern, by far the most common [11]. Contrary to previous perceptions, ILD is found almost as frequently in limited compared to diffuse skin disease, and the rate of FVC decline does not appear to differ between the two subsets [12].

ILD in the context of SSc can be asymptomatic and patients may first present with extensive lung disease. On the other hand, most patients with SSc have limited ILD involvement, which will have a stable course even without treatment. The reliable detection of patients at risk of lung disease progression is particularly difficult in patients with mild, early disease. The identification of non-invasive, prognostic biomarkers that predict the likelihood of disease progression has the potential to allow timely immunosuppressant therapy, while avoiding unnecessary treatment in patients likely to follow an indolent course. Furthermore, identification of patients more at risk of disease progression is needed for cohort enrichment in randomised controlled clinical trials, which have so far included a proportion of patients with stable SSc-ILD, thus potentially diluting the effects of the intervention [13].

Awareness of potential confounding factors is crucial to correctly interpret biomarker associations with SSc-ILD. In the context of SSc, serum biomarkers can reflect extrapulmonary disease activity, including rapidly progressive diffuse skin fibrosis and active systemic disease. From the pulmonary aspect, DLCO is a sensitive marker of ILD, but also reflects pulmonary vasculopathy. Indeed, a disproportionate reduction in DLCO in relation to lung volumes should prompt the assessment of pulmonary hypertension
[14]. Adjustment for these factors in multivariate analysis is crucial to assess the correlation between the putative biomarkers and SSc-ILD.

Biomarkers can fulfil a number of roles, including identification of pathways involved in disease pathogenesis, assessment of disease severity, prediction of future disease behaviour and use as surrogate outcome measures [15]. This paper focuses on peripheral blood biomarkers, as they offer a number of advantages - they are readily obtained, can be measured longitudinally, and thus have the potential to be integrated into clinical use.

\section{Autoantibody Subsets}

Autoantibody subsets have strong associations with different patterns of pulmonary involvement. Anti-topoisomerase I antibodies (ATA), have been consistently associated with pulmonary fibrosis, while anti-centromere antibodies (ACA) are linked to pulmonary hypertension and are rarely present in SSc-ILD [16]. While it is clear that ATA positivity is associated with a greater risk of lung fibrosis, it remains unclear whether it is associated with more progressive ILD. A reduced incidence of lung fibrosis is found in antiRNA polymerase III positive patients [17]. The nucleolar autoantibodies, anti-U3 RNP antibody and anti TH/To, are linked to an increased risk of pulmonary disease and appear to be associated with development of pulmonary hypertension disproportionate to the degree of interstitial involvement [18].

\section{Alveolar Epithelial Proteins}

Although the sequence of events in the pathogenesis of SSc-ILD is not fully understood, ultrastructural studies have shown that both epithelial and endothelial cell 
injury may precede inflammation and fibrosis [19]. The increased clearance of technetium-labeled diethylene triamine pentaacetate (99mTc-DTPA) aerosol from the lungs of patients with SSc-ILD is indicative of a breached epithelial barrier and is associated with more rapid decline in FVC $[20,21]$, independently of disease severity. Lung epithelium-specific proteins leaking into the circulation may thus represent potential biomarkers of alveolar epithelial damage.

3.1. Surfactant Proteins ( $S P-A$ and $S P-D)$. Secreted by type II alveolar epithelial cells and airway Clara cells, pulmonary surfactants are lipoprotein complexes that include the hydrophobic proteins SP-B and SP-C and hydrophilic proteins SP-A and SP-D [22]. In addition to stabilising alveolar surface tension, they play an important role in the lung's host defence system, a role mediated primarily by SPA and SP-D.

Serum levels of SP-A and SP-D are significantly higher in SSc patients with pulmonary fibrosis than in those without $[23,24]$, and SP-D serum levels are negatively correlated with VC and DLCO $[24,25]$. SP-D levels are more sensitive than SP-A in detecting ILD as defined by CT. Using cut-off levels set at $43.8 \mathrm{ng} / \mathrm{mL}$ for SP-A and $110 \mathrm{ng} / \mathrm{mL}$ for SP-D, the sensitivities and specificities for detecting CT-positive ILD in 42 patients with SSc were $33 \%$ and $100 \%$ for SP-A and $77 \%$ and $83 \%$ for SP-D, respectively [23]. Receiver operator curve analysis demonstrated good sensitivity $(89.4 \%)$ and specificity (80\%) of SP-D levels $>90 \mathrm{ng} / \mathrm{mL}$ in the assessment of "alveolitis" as defined by BAL neutrophilia and/or HRCT ground glass in the Scleroderma Lung Study [26]. In a small but prospective study of 35 patients with SSc-ILD followed over 1-10 years, SP-D levels were seen to definitely increase over time in 9 out of the 10 patients with worsening ILD, as defined by changes in symptoms, lung function, and imaging, compared to mild increases in only 3 out of 25 patients with stable or improving SSc-ILD [24]. In a small, retrospective study of 6 patients with SSc-ILD by Yanaba et al., serum SP-D levels were analysed over a mean followup period of 2.3 years. In 3 out of the 4 patients treated with immunosuppressive therapy, longitudinal SP-D levels decreased/stabilised in parallel with lung function findings [25].

3.2. Krebs Von Den Lungen 6 (KL-6). KL-6 is a high-molecular-weight mucin-like glycoprotein, strongly expressed by type II alveolar epithelial cells and bronchiolar epithelial cells [27], which increases following cellular injury and/or regeneration. Additionally, KL-6 has been shown to have profibrotic and antiapoptotic effects on lung fibroblasts and thus may have a role in the pathogenesis of SSc-ILD [28].

Serum KL-6 is elevated in a variety of different ILDs, including the idiopathic interstitial pneumonias, hypersensitivity pneumonitis, drug-induced pneumonitis, sarcoidosis, and connective tissue disease-associated ILD (CTD-ILD), reflecting the prominent role of alveolar epithelial injury and proliferation across ILDs [29]. Serum levels of KL6 are significantly higher in SSc patients with pulmonary fibrosis than in SSc alone, and levels inversely correlate with VC and DLCO $[25,30]$. A threshold of $500 \mathrm{U} / \mathrm{mL}$, commonly used also in the context of other interstitial lung diseases, provided a $78.8 \%$ sensitivity and $90 \%$ specificity in detecting "alveolitis" as defined in the Scleroderma Lung Study [26]. In a study of 240 patients with a variety of CTDs, a serum level $>500 \mathrm{U} / \mathrm{mL}$ was a marker of ILD (sensitivity 0.79 ; specificity 0.93 ; diagnostic accuracy 0.896 ), and a level $>1000 \mathrm{U} / \mathrm{mL}$ was a marker of "active" progressive ILD (sensitivity 0.867 ; specificity 0.865 ; diagnostic accuracy 0.866 ), although this was loosely defined as disease requiring newly added intervention [31]. Bonella et al. found a stronger correlation for KL-6 than SP-D with HRCT fibrosis scores and confirmed the described association with FVC and DLCO [32].

In a retrospective longitudinal study, KL-6 levels were determined in 39 patients with SSc during a follow-up period of 0.3-6.1 years [33]. New onset or deterioration of ILD occurred in 4 patients and was associated with a rapid increase in serum KL-6 levels. Reflecting its potential use in serial monitoring of disease activity, levels of KL-6 have been shown to decrease after treatment with cyclophosphamide [34]. Satoh et al. evaluated the prognostic significance of serum KL-6 levels in 219 patients with ILD, including 67 with CTD-ILD. Higher levels of KL-6 were seen in patients who died during the follow-up period, with a threshold level of $1000 \mathrm{U} / \mathrm{mL}$ discriminating nonsurvivors from survivors [35].

Overall, KL-6 appears to be a promising multipurpose biomarker that appears to reflect response to treatment and prognosis in SSc-ILD. Although integrated into clinical practice in Japan for a number of years, it still requires validation in a large number of patients with SSc-ILD, particularly to assess whether it can predict outcome independently of markers of ILD severity.

\section{CC and CXC Chemokines}

Chemokines traffic leucocytes to sites of inflammation and are classified into subsets based on the position of their first two cysteine residues: CC and CXC chemokines. Both bind to receptors expressed on the surface of leucocytes, CXCR and CCR, antagonists of which may represent candidates for targeted therapy.

4.1. CC Chemokine Ligand 18 (CCL18). CCL18, previously known as pulmonary and activation-regulated chemokine (PARC), is constitutively expressed at high levels in the lungs, mainly by alveolar macrophages, and acts as a chemoattractant for a variety of mononuclear cells. CCL18 production by alveolar macrophages, alternatively activated by Th2 cytokines, is increased in a number of fibrotic lung diseases, including SSc-ILD, and correlates with serum levels [36]. CCL18 has been shown to stimulate fibroblast collagen synthesis [37], which in turn appears to further increase CCL18 production [38].

In SSc, serum CCL18 levels are inversely correlated with baseline lung function and have been associated with 
longitudinal changes in VC and DLCO [36, 39]. In a longitudinal analysis of 21 patients with SSc-ILD by Kodera et al., serum CCL18 correlated with ILD activity (determined by HRCT, lung function, and BAL analysis), possibly more tightly than KL-6 or SP-D. In a recent, prospective cohort study of 83 SSc patients over a four-year period by Tiev et al., increased serum CCL18 levels were independently predictive of ILD worsening [40]. A baseline CCL18 level >187 ng/mL was the best cut-off (53\% sensitivity; 96\% specificity) for identifying subsequent lung function worsening $(10 \%$ decrease from baseline of TLC or FVC) or death, with a hazard ratio of 5.36 (95\% CI 2.44-11.75; $P=0.001)$, even after adjustment for baseline DLCO and duration of disease [40]. To our knowledge this is the first large study to prospectively evaluate the prognostic ability of a biomarker in SSc-ILD, adjusting for ILD severity and other covariates. Interestingly, CCL18 has also been prospectively evaluated in patients with idiopathic pulmonary fibrosis by Prasse et al. and was found to predict early lung function decline and mortality, with a similar cut-off level of $150 \mathrm{ng} / \mathrm{mL}$, again independently of disease severity [41], suggesting its utility as a marker of ILD progression independently of the setting. Overall, if confirmed by separate prospective studies, these results suggest that serum CCL18 could be used in clinical management as a marker of progressive ILD, to aid in targeting treatment to the correct patients, and should be evaluated as a potential therapeutic target.

4.2. CC Chemokine Ligand 2 (CCL2). CCL2, also known as monocyte chemoattractant protein-1 (MCP-1), is chemoattractant for monocytes and $\mathrm{T}$ cells and has been shown to induce Th2 cell polarisation, thereby stimulating collagen production and myofibroblast differentiation in fibroblasts $[42,43]$. Serum levels are upregulated in SSc and correlate with the presence of ILD [44-46]. In a study of 33 SSc patients, serum CCL2 variation correlated with changes in VC during a 3-year follow-up period [47], although the number of patients with declining lung function was small. Bronchoalveolar lavage CCL2 concentration was associated with the presence of ILD in 32 SSc patients and correlated with lung function parameters and CT scores [48].

4.3. CXC Chemokine Ligand 10 (CXCL10). CXCL10 displays strong chemoattractant activity for Th1 lymphocytes with elevated serum levels seen in various autoimmune diseases [49]. In SSc, serum levels are significantly increased in the presence of ILD compared to those without and normal controls $[46,50]$. However, a recent retrospective, longitudinal study of 31 SSc patients found that levels did not correlate with change in lung function over time [47].

4.4. CXC Chemokine Ligand 12 (CXCL12). The expression of CXCL12 and its receptor CXCR4 is critical for the recruitment of circulating progenitor cells during tissue repair. Circulating CXCR4+ progenitor cells have been observed to correlate with skin and lung involvement [51] and are found in the lung tissue of patients with SSc-ILD but not in controls, with parallel upregulation of its ligand
CXCL12, expressed by alveolar epithelial cells and alveolar macrophages [52].

\section{Leukocytes}

5.1. T-Cell Subsets. Two functional subsets of $\mathrm{T}$ cells with distinct cytokine-secretion profiles are well recognised [53]. Type 1 (Th1) T cells predominantly produce interleukin-2 (IL-2) and interferon-gamma (IFN- $\gamma$ ), whereas type 2 (Th2) T cells produce interleukins IL-4, IL-5, IL-6, IL-10, and IL-13 [54]. These subsets appear to exert opposing roles in tissue remodelling and fibrogenesis; IFN- $\gamma$ suppresses fibroblast activity whereas the Th2 cytokines such as IL-4, IL-6, and IL-13 are profibrotic either by directly stimulating collagen synthesis by fibroblasts or indirectly by inducing profibrotic cytokines such as transforming growth factor-beta (TGF- $\beta$ ) and connective tissue growth factor (CTGF) [55].

In patients with $\mathrm{SSc}$, a predominant Th2 cytokine profile has been reported in lung tissue [56], bronchoalveolar lavage fluid [57], and peripheral blood [56]. In SSc-ILD, a further marked reduction in the $\mathrm{Th} 1 / \mathrm{Th} 2$ ratio compared to SSc patients without ILD has been reported, with a strong linear correlation between a lower Th1/Th2 ratio and lower FVC levels [56]. Serum levels of IL-10 and IL-6 have been found to be elevated in patients with SSc-ILD compared to SSc alone $[47,58]$, and our group has recently shown that elevated IL6 is independently associated with lung function worsening and increased mortality in patients with SSc-ILD (submitted for publication). Serum levels of IL-15, a pleiotropic cytokine which may have a pathogenetic role in both fibrotic and vascular lung disease, were found to strongly correlate with impaired lung function in SSc [59].

Th17 lymphocytes, recently described as a subset which synthesise an array of cytokines including IL-17A, are the main producers of TGF- $\beta$ among Th subsets [60]. Increased Th17 cells and IL-17A levels have been detected in the sera, skin, and lungs of patients with SSc [61-63], although another study reported normal serum IL-17A levels in SSc [64]. Th17 is induced by various cytokines including IL-23, IL-6, and TGF- $\beta$; interestingly, IL-23 has also been associated with the presence of ILD in SSc [65]. IL-22 is a cytokine which plays a role in the maintenance and integrity of epithelial barrier function [66], produced by Th17 and Th22 lymphocytes, another novel T-cell subset [67]. Circulating IL-22- and IL-17-producing T cells were increased in SSc individuals presenting with ILD, as detected by HRCT and decreased TLC, compared with those without ILD [68].

5.2. B Cells. B-cell infiltration has been recently demonstrated in SSc lung and skin [69, 70]. There is evidence of circulating B cell activation in SSc [71] with overexpression of CD19, a positive regulator that increases B-cell signals in response to antigens [72]. In patients with SSc-ILD, Bcell-depleting therapy with Rituximab significantly improved lung function in a small, randomised, controlled study by Daoussis et al. [73], suggesting a crucial role of Bcells in the progression of fibrosis in SSc. These results suggest circulating B-cell markers should be assessed in 
relation to lung involvement, as they may ultimately identify subsets more likely to respond to B-cell depletion treatment strategies.

\section{Macrophages/Monocytes}

Macrophages, mostly derived from CD14+ monocytes, can display two distinct phenotypes of activation: Th1 or "classically" activated (M1) macrophages arise in response to IFN- $\gamma$ or IL-1 whereas Th2, or "alternatively" activated (M2), macrophages are derived following exposure to Th2 cytokines, including IL-4, IL-13, and IL-10 [74], and are characterised by an anti-inflammatory and profibrotic phenotype. The CD14+ monocyte fraction from peripheral blood in patients with SSc-ILD is characterised by substantially increased expression of the activation marker CD163, which colocalises with the M2 marker CD204 [75], and, following stimulation with lipopolysaccharide, release of the profibrotic mediators CCL18 and IL-10 [76], characteristic products of M2 macrophages. There also exists a population of CD14+/CD45+/collagen I-producing circulating monocytes which are increased in the peripheral circulation of patients with SSc-ILD compared to controls [76].

\section{Matrix Metalloproteinases/Tissue Inhibitors of Metalloproteinases}

Remodelling of the extracellular matrix and maintenance of basement membrane integrity involve a balance between the matrix metalloproteinases (MMPs) and their inhibitors, tissue inhibitors of metalloproteinases (TIMPs). MMP-7 (matrilysin), a metalloproteinase which targets a broad range of extracellular matrix proteins, was originally found to be highly overexpressed in IPF lungs [77] and subsequently in other interstitial lung diseases [78]. In IPF, both BAL and serum MMP-7 are negatively correlated with FVC and DLCO [79]. Among SSc patients, higher levels of serum MMP-7 were seen in patients with lung fibrosis compared to those without and were associated with lower DLCO levels, although the association of MMP-7 levels with ILD progression was not evaluated [80]. Notably, patients with lung fibrosis and concomitant pulmonary hypertension had higher mean MMP-7 serum levels compared to those with lung fibrosis alone, indicative of the potential confounding issue of underlying vasculopathy in DLCO reduction.

MMP-9 (gelatinase B), associated with chronic inflammatory autoimmune diseases and conditions characterised by excessive fibrosis, was significantly increased in serum of SSc patients compared to healthy controls [81] and in bronchoalveolar lavage of SSc-ILD patients [82]. Interestingly, circulating levels of MMP-12 have been reported to be tightly inversely correlated with FVC $(r=-0.82)$, and MMP-12 staining to be significantly increased in SSc-ILD lungs compared to normal controls [83]. TIMP-1 levels have been shown to correlate with the presence of ILD in SSc and to negatively correlate with DLCO, albeit weakly $(r=-0.28)$ [84].

\section{Neutrophil Elastase}

Polymorphonuclear neutrophilic leukocyte (PMN) elastase is a serine proteinase that is believed to modulate extracellular matrix formation and remodelling following lung injury [85]. As elegantly reviewed by Hant and Silver, serum levels of PMN elastase have been reported as significantly increased in SSc-ILD and interestingly correlated well with SP-D and KL-6 $[15,86]$.

\section{Profibrotic Growth Factors}

Serum connective tissue growth factor (CTGF) has been found to be increased in SSc patients, and to correlate with skin and lung fibrosis $[87,88]$, although longitudinal studies to evaluate prognostic ability have not been performed. Interestingly, serum TGF- $\beta$, considered one of the master regulators of tissue fibrosis, is not consistently upregulated in SSc patients and indeed was found to be reduced in patients with active diffuse skin disease, perhaps reflecting sequestration to active SSc skin disease [89].

\section{Markers of Oxidative Stress}

Oxidative stress mediated by free oxygen radicals is believed to play a role in the pathogenesis of lung fibrosis and of systemic sclerosis. Serum total antioxidant power, a measure of global antioxidant activity, was found to be increased in SSc patients, although did not differ according to presence of ILD [90]. By contrast, serum isoprostane, a marker of lipid peroxidation, was associated with SSc-ILD and was inversely correlated with FVC and DLCO ( $r=-0.4$ for both) [91]. Urinary levels of F2-isoprostanes, generated by free radical peroxidation of arachidonic acid, also inversely correlated with DLCO $(r=-0.44)$. Although the association with ILD severity is interesting, none of the oxidative stress markers were assessed as potential predictors of ILD progression.

\section{Acute-Phase Proteins}

Acute-phase reactant proteins are elevated in a proportion of patients with SSc. An elevated erythrocyte sedimentation rate (ESR) has been shown to be independently associated with mortality in a number of studies [92, 93]. C-reactive protein (CRP) significantly correlates with ESR [94] and with serum IL-6 in SSc [95]. In 1043 SSc patients, CRP was observed to correlate with disease severity, poor pulmonary function, and shorter survival [94]. Sharing a C-terminal pentraxin domain with CRP, pentraxin 3 (PTX3), an acutephase protein produced at disease sites by a number of cell types, including fibroblasts [96], was found to correlate with lung function impairment in SSc [97].

\section{Vitamin D}

Insufficient levels of vitamin $\mathrm{D}$ have been reported in a number of autoimmune diseases [98], including SSc [99]. In a study of 327 patients with SSc, vitamin D deficiency 
was associated with reduced DLCO $(P<0.02)[100]$. An association with DLCO and/or increased pulmonary artery pressures on echocardiography had also been reported in two smaller earlier studies [99, 101], suggesting a possible correlation with pulmonary vasculopathy, rather than ILD.

\section{Endothelial Cell Activation}

Ultrastructural studies have shown that both epithelial and endothelial cell injury precede inflammation and fibrosis [19]. Following endothelial activation, a procoagulant and profibrotic environment ensues. Endothelin-1 (ET-1), an endothelial cell product with well-known vasoconstrictor properties, has also been shown to promote profibrotic processes, including the induction of myofibroblast differentiation, contraction, and extracellular matrix synthesis [102]. Increased ET-1 levels have been found in BAL fluid and lung tissue from patients with SSc $[103,104]$. Increased serum levels of soluble vascular cell adhesion molecule1 (sVCAM-1), soluble E-selectin (sE-selectin), vascular endothelial growth factor (VEGF), and endothelin-1 (ET1) have been described in patients with SSc, and have been variably correlated with internal organ involvement, including pulmonary fibrosis [105-107]. Anti-endothelial cell antibodies (AECA), postulated to play a role in vascular injury in a number of autoimmune diseases, have been reported in $22 \%-86 \%$ of patients with SSc, depending on detection methods used [108], and have been associated with a higher frequency of pulmonary fibrosis [109].

\section{Serum Biomarkers of Pulmonary Hypertension in SSc-ILD}

A detailed analysis of pulmonary hypertension markers in SSc is outside of the scope of this paper. Pulmonary hypertension in SSc (SSc-PH) can occur in the absence of interstitial lung involvement, in association with ILD and/or as a consequence of left-sided heart disease. Compared to patients with SSc-PH alone, those with SSc-ILD associated $\mathrm{PH}$ have a worse prognosis, with a recently reported 3year survival of $71 \%$ versus $47 \%$, respectively $(P=0.07)$ [110]. Indicators suggestive of the development of $\mathrm{PH}$ in the context of ILD include a disproportionate reduction in DLCO compared to lung volumes and development of hypoxia $[111,112]$. Echocardiography is used as a screening tool, but in patients with ILD is plagued by low specificity [113]. Although a right-sided cardiac catheterisation remains the gold standard for the diagnosis of $\mathrm{PH}$, the procedure is invasive and cannot be used routinely for screening/monitoring purposes.

Among serum biomarkers, natriuretic peptides, including brain natriuretic peptide (BNP) and N-terminal pro-brain natriuretic peptide (NT-pro-BNP), released in response to ventricular stretch by cardiomyocytes, have been established as informative markers of RV dysfunction in $\mathrm{PH}[114,115]$. BNP was found to be a marker of poor prognosis in patients with chronic lung diseases [116]. In a study of 90 ILD patients by Corte et al., including 18 with
CTD-ILD, serum BNP correlated with echocardiographic estimates of pulmonary pressures. Furthermore, a serum $\mathrm{BNP} \geq 20 \mathrm{pmol} \cdot \mathrm{L}^{-1}$ was associated with a 14 -fold increased mortality compared to patients with $\mathrm{BNP}<4 \mathrm{pmol} \cdot \mathrm{L}^{-1}$ [117]. However, natriuretic peptide levels only rise when there is strain to the right ventricle, and markers of the earlier stages of pulmonary vasculopathy are needed to allow early intervention.

\section{Conclusion}

The majority of the studies performed to date have identified a link between serum proteins and the presence or severity of ILD in the context of SSc. In a disease with extremely variable presentation and outcome, there is an unmet clinical need for biomarkers predictive of ILD progression over time, independently of disease severity. In particular, clinicians need a biomarker to target patients with early and limited ILD at higher risk of disease progression for early therapy, so as to prevent the development of extensive disease. Ideally, a biomarker would also provide early assessment of response to treatment and could theoretically identify subsets of SSc-ILD with differential response to targeted therapies. In providing information on the likelihood of future ILD progression, biomarkers should also allow better selection of SSc-ILD patients for clinical trials, to enrich trial cohorts with patients more at risk of disease progression.

To date, there have been very few studies evaluating the ability of biomarkers to predict SSc-ILD progression. The most promising biomarkers thus far appear to be KL-6 and CCL18, and the evidence for their utilisation in predicting likelihood of progression of SSc-ILD and monitoring treatment response is encouraging. However, the panel of investigated biomarkers so far has been limited. In idiopathic pulmonary fibrosis, prospective, multicentre, longitudinal trials are underway to identify if a broad array of biological biomarkers collected at the time of diagnosis and at various longitudinal time points can be used to predict the disease course-COMET: correlating outcomes with biochemical markers to estimate time-progression in IPF (NCT01071707) and PROFILE: prospective observation of fibrosis in the lung clinical endpoints (NCT01110694). A similar endeavour is yet to be undertaken in SSc-ILD.

Prospective, well-designed studies, with detailed characterisation of ILD at baseline, to allow adjustment for disease severity and meticulous monitoring of disease progression outcomes and serum biomarkers are needed. The use of high throughput gene expression, protein and immune serum biomarker profiling is likely to identify a combination of biomarkers, each expressing different pathogenetic pathways, that when combined may provide a powerful indication of the likelihood of ILD progression and allow early evaluation of response to treatment.

\section{Acknowledgment}

The authors acknowledge the support of the Raynaud's and Scleroderma Association. 


\section{References}

[1] V. D. Steen and T. A. Medsger Jr., "Changes in causes of death in systemic sclerosis, 1972-2002," Annals of the Rheumatic Diseases, vol. 66, no. 7, pp. 940-944, 2007.

[2] A. J. Tyndall, B. Bannert, M. Vonk et al., "Causes and risk factors for death in systemic sclerosis: a study from the EULAR Scleroderma Trials and Research (EUSTAR) database," Annals of the Rheumatic Diseases, vol. 69, no. 10, pp. 1809-1815, 2010.

[3] E. A. Renzoni, "Interstitial lung disease in systemic sclerosis," Monaldi Archives for Chest Disease, vol. 67, no. 4, pp. 217-228, 2007.

[4] V. D. Steen and T. A. Medsger Jr., "Severe organ involvement in systemic sclerosis with diffuse scleroderma," Arthritis and Rheumatism, vol. 43, no. 11, pp. 2437-2444, 2000.

[5] C. Morgan, C. Knight, M. Lunt, C. M. Black, and A. J. Silman, "Predictors of end stage lung disease in a cohort of patients with scleroderma," Annals of the Rheumatic Diseases, vol. 62, no. 2, pp. 146-150, 2003.

[6] A. U. Wells, D. M. Hansell, M. B. Rubens et al., "Fibrosing alveolitis in systemic sclerosis: indices of lung function in relation to extent of disease on computed tomography," Arthritis and Rheumatism, vol. 40, no. 7, pp. 1229-1236, 1997.

[7] D. P. Tashkin, R. Elashoff, P. J. Clements et al., "Cyclophosphamide versus placebo in scleroderma lung disease," The New England Journal of Medicine, vol. 354, no. 25, pp. 2655 2666, 2006.

[8] N. S. L. Goh, S. R. Desai, S. Veeraraghavan et al., "Interstitial lung disease in systemic sclerosis: a simple staging system," American Journal of Respiratory and Critical Care Medicine, vol. 177, no. 11, pp. 1248-1254, 2008.

[9] N. S. L. Goh, S. Veeraraghavan, S. R. Desai et al., "Bronchoalveolar lavage cellular profiles in patients with systemic sclerosis-associated interstitial lung disease are not predictive of disease progression," Arthritis and Rheumatism, vol. 56, no. 6, pp. 2005-2012, 2007.

[10] C. Strange and J. R. Seibold, "Scleroderma lung disease: 'If you don't know where you are going, any road will take you there," American Journal of Respiratory and Critical Care Medicine, vol. 177, no. 11, pp. 1178-1179, 2008.

[11] D. Bouros, A. U. Wells, A. G. Nicholson et al., "Histopathologic subsets of fibrosing alveolitis in patients with systemic sclerosis and their relationship to outcome," American Journal of Respiratory and Critical Care Medicine, vol. 165, no. 12, pp. 1581-1586, 2002.

[12] P. J. Clements, M. D. Roth, R. Elashoff et al., "Scleroderma lung study (SLS): differences in the presentation and course of patients with limited versus diffuse systemic sclerosis," Annals of the Rheumatic Diseases, vol. 66, no. 12, pp. 16411647, 2007.

[13] D. Khanna, K. K. Brown, P. J. Clements et al., "Systemic sclerosis-associated interstitial lung disease-proposed recommendations for future randomised clinical trials," Clinical and Experimental Rheumatology, vol. 28, no. 2, supplement 58, pp. S55-S62, 2010.

[14] A. De Lauretis, S. Veeraraghavan, and E. Renzoni, "Connective tissue disease-associated interstitial lung disease: how does it differ from IPF? How should the clinical approach differ?" Chronic Respiratory Disease, vol. 8, no. 1, pp. 53-82, 2011.

[15] F. N. Hant and R. M. Silver, "Biomarkers of scleroderma lung disease: recent progress," Current Rheumatology Reports, vol. 13, no. 1, pp. 44-50, 2011.
[16] V. D. Steen, D. L. Powell, and T. A. Medsger Jr., "Clinical correlations and prognosis based on serum autoantibodies in patients with systemic sclerosis," Arthritis and Rheumatism, vol. 31, no. 2, pp. 196-203, 1988.

[17] Y. Okano, V. D. Steen, and T. A. Medsger Jr., "Autoantibody reactive with RNA polymerase III in systemic sclerosis," Annals of Internal Medicine, vol. 119, no. 10, pp. 1005-1013, 1993.

[18] V. Steen, "Predictors of end stage lung disease in systemic sclerosis," Annals of the Rheumatic Diseases, vol. 62, no. 2, pp. 97-99, 2003.

[19] N. K. Harrison, A. R. Myers, B. Corrin et al., "Structural features of interstitial lung disease in systemic sclerosis," American Review of Respiratory Disease, vol. 144, no. 3, pp. 706-713, 1991.

[20] A. U. Wells, D. M. Hansell, N. K. Harrison, R. Lawrence, C. M. Black, and R. M. Du Bois, "Clearance of inhaled 99mTcDTPA predicts the clinical course of fibrosing alveolitis," European Respiratory Journal, vol. 6, no. 6, pp. 797-802, 1993.

[21] N. S. L. Goh, S. R. Desai, C. Anagnostopoulos et al., "Increased epithelial permeability in pulmonary fibrosis in relation to disease progression," European Respiratory Journal, vol. 38, no. 1, pp. 184-190, 2011.

[22] A. M. Pastva, J. R. Wright, and K. L. Williams, "Immunomodulatory roles of surfactant proteins A and D: implications in lung disease," Proceedings of the American Thoracic Society, vol. 4, no. 3, pp. 252-257, 2007.

[23] H. Takahashi, Y. Kuroki, H. Tanaka et al., "Serum levels of surfactant proteins A and D are useful biomarkers for interstitial lung disease in patients with progressive systemic sclerosis," American Journal of Respiratory and Critical Care Medicine, vol. 162, no. 1, pp. 258-263, 2000.

[24] Y. Asano, H. Ihn, K. Yamane et al., "Clinical significance of surfactant protein D as a serum marker for evaluating pulmonary fibrosis in patients with systemic sclerosis," Arthritis and Rheumatism, vol. 44, no. 6, pp. 1363-1369, 2001.

[25] K. Yanaba, M. Hasegawa, K. Takehara, and S. Sato, "Comparative study of serum surfactant protein-D and KL-6 concentrations in patients with systemic sclerosis as markers for monitoring the activity of pulmonary fibrosis," Journal of Rheumatology, vol. 31, no. 6, pp. 1112-1120, 2004.

[26] F. N. Hant, A. Ludwicka-Bradley, H. J. Wang et al., "Surfactant protein D and KL-6 as serum biomarkers of interstitial lung disease in patients with scleroderma," Journal of Rheumatology, vol. 36, no. 4, pp. 773-780, 2009.

[27] N. Kohno, S. Kyoizumi, Y. Awaya, H. Fukuhara, M. Yamakido, and M. Akiyama, "New serum indicator of interstitial pneumonitis activity. Sialylated carbohydrate antigen KL-6," Chest, vol. 96, no. 1, pp. 68-73, 1989.

[28] S. Ohshimo, A. Yokoyama, N. Hattori, N. Ishikawa, Y. Hirasawa, and N. Kohno, "KL-6, a human MUC1 mucin, promotes proliferation and survival of lung fibroblasts," Biochemical and Biophysical Research Communications, vol. 338, no. 4, pp. 1845-1852, 2005.

[29] N. Ishikawa, N. Hattori, A. Yokoyama, and N. Kohno, "Utility of KL-6/MUC1 in the clinical management of interstitial lung diseases," Respiratory Investigation, vol. 50, no. 1, pp. 3-13, 2012.

[30] K. Yamane, H. Ihn, M. Kubo et al., "Serum levels of KL-6 as a useful marker for evaluating pulmonary fibrosis in patients with systemic sclerosis," Journal of Rheumatology, vol. 27, no. 4, pp. 930-934, 2000.

[31] S. Doishita, S. Inokuma, H. Asashima et al., "Serum KL6 level as an indicator of active or inactive interstitial 
pneumonitis associated with connective tissue diseases," Internal Medicine, vol. 50, no. 23, pp. 2889-2892, 2011.

[32] F. Bonella, A. Volpe, P. Caramaschi et al., "Surfactant protein D and KL-6 serum levels in systemic sclerosis: correlation with lung and systemic involvement," Sarcoidosis Vasculitis and Diffuse Lung Diseases, vol. 28, no. 1, pp. 27-33, 2011.

[33] K. Yanaba, M. Hasegawa, Y. Hamaguchi, M. Fujimoto, K. Takehara, and S. Sato, "Longitudinal analysis of serum KL6 levels in patients with systemic sclerosis: association with the activity of pulmonary fibrosis," Clinical and Experimental Rheumatology, vol. 21, no. 4, pp. 429-436, 2003.

[34] M. Okada, K. Suzuki, M. Matsumoto et al., "Intermittent intravenous cyclophosphamide pulse therapy for the treatment of active interstitial lung disease associated with collagen vascular diseases," Modern Rheumatology, vol. 17, no. 2, pp. 131-136, 2007.

[35] H. Satoh, K. Kurishima, H. Ishikawa, and M. Ohtsuka, "Increased levels of KL-6 and subsequent mortality in patients with interstitial lung diseases," Journal of Internal Medicine, vol. 260, no. 5, pp. 429-434, 2006.

[36] A. Prasse, D. V. Pechkovsky, G. B. Toews et al., "CCL18 as an indicator of pulmonary fibrotic activity in idiopathic interstitial pneumonias and systemic sclerosis," Arthritis and Rheumatism, vol. 56, no. 5, pp. 1685-1693, 2007.

[37] S. P. Atamas, I. G. Luzina, J. Choi et al., "Pulmonary and activation-regulated chemokine stimulates collagen production in lung fibroblasts," American Journal of Respiratory Cell and Molecular Biology, vol. 29, no. 6, pp. 743-749, 2003.

[38] A. Prasse, D. V. Pechkovsky, G. B. Toews et al., "A vicious circle of alveolar macrophages and fibroblasts perpetuates pulmonary fibrosis via CCL18," American Journal of Respiratory and Critical Care Medicine, vol. 173, no. 7, pp. 781-792, 2006.

[39] M. Kodera, M. Hasegawa, K. Komura, K. Yanaba, K. Takehara, and S. Sato, "Serum pulmonary and activationregulated chemokine/CCL18 levels in patients with systemic sclerosis: a sensitive indicator of active pulmonary fibrosis," Arthritis and Rheumatism, vol. 52, no. 9, pp. 2889-2896, 2005.

[40] K. P. Tiev, T. Hua-Huy, A. Kettaneh et al., "Serum CC chemokine ligand-18 predicts lung disease worsening in systemic sclerosis," European Respiratory Journal, vol. 38, no. 6, pp. 1355-1360, 2011.

[41] A. Prasse, C. Probst, E. Bargagli et al., "Serum CC-chemokine ligand 18 concentration predicts outcome in idiopathic pulmonary fibrosis," American Journal of Respiratory and Critical Care Medicine, vol. 179, no. 8, pp. 717-723, 2009.

[42] M. T. Carulli, V. H. Ong, M. Ponticos et al., "Chemokine receptor CCR2 expression by systemic sclerosis fibroblasts: evidence for autocrine regulation of myofibroblast differentiation," Arthritis and Rheumatism, vol. 52, no. 12, pp. 3772 3782, 2005.

[43] L. Gu, S. Tseng, R. M. Horner, C. Tam, M. Loda, and B. J. Rollins, "Control of $\mathrm{T}(\mathrm{H}) 2$ polarization by the chemokine monocyte chemoattractant protein-1," Nature, vol. 404, no. 6776, pp. 407-411, 2000.

[44] M. T. Carulli, C. Handler, J. G. Coghlan, C. M. Black, and C. P. Denton, "Can CCL2 serum levels be used in risk stratification or to monitor treatment response in systemic sclerosis?" Annals of the Rheumatic Diseases, vol. 67, no. 1, pp. 105-109, 2008.

[45] M. Hasegawa, S. Sato, and K. Takehara, "Augmented production of chemokines (monocyte chemotactic protein-1 (MCP-1), macrophage inflammatory protein-1 $\alpha$ (MIP-1 $\alpha$ ) and MIP-1 $\beta$ ) in patients with systemic sclerosis: MCP-1 and MIP- $1 \alpha$ may be involved in the development of pulmonary fibrosis," Clinical and Experimental Immunology, vol. 117, no. 1, pp. 159-165, 1999.

[46] A. Antonelli, C. Ferri, P. Fallahi et al., "CXCL10 $(\alpha)$ and CCL2 $(\beta)$ chemokines in systemic sclerosis—a longitudinal study," Rheumatology, vol. 47, no. 1, pp. 45-49, 2008.

[47] M. Hasegawa, M. Fujimoto, T. Matsushita, Y. Hamaguchi, K. Takehara, and S. Sato, "Serum chemokine and cytokine levels as indicators of disease activity in patients with systemic sclerosis," Clinical Rheumatology, vol. 30, no. 2, pp. 231-237, 2011.

[48] K. Schmidt, L. Martinez-Gamboa, S. Meier et al., "Bronchoalveoloar lavage fluid cytokines and chemokines as markers and predictors for the outcome of interstitial lung disease in systemic sclerosis patients," Arthritis Research \& Therapy, vol. 11, no. 4, article R111, 2009.

[49] E. Y. Lee, Z. H. Lee, and Y. W. Song, "CXCL10 and autoimmune diseases," Autoimmunity Reviews, vol. 8, no. 5, pp. 379383, 2009.

[50] H. Fujii, Y. Shimada, M. Hasegawa, K. Takehara, and S. Sato, "Serum levels of a Th1 chemoattractant IP-10 and Th2 chemoattractants, TARC and MDC, are elevated in patients with systemic sclerosis," Journal of Dermatological Science, vol. 35, no. 1, pp. 43-51, 2004.

[51] D. Campioni, A. Lo Monaco, F. Lanza et al., "CXCR4pos circulating progenitor cells coexpressing monocytic and endothelial markers correlating with fibrotic clinical features are present in the peripheral blood of patients affected by systemic sclerosis," Haematologica, vol. 93, no. 8, pp. 12331237, 2008.

[52] E. Tourkina, M. Bonner, J. Oates et al., "Altered monocyte and fibrocyte phenotype and function in scleroderma interstitial lung disease: reversal by caveolin-1 scaffolding domain peptide," Fibrogenesis and Tissue Repair, vol. 4, no. 1, article 15, 2011.

[53] S. Romagnani, "The Th1/Th2 paradigm," Immunology Today, vol. 18, no. 6, pp. 263-266, 1997.

[54] T. R. Mosmann and R. L. Coffman, "TH1 and TH2 cells: different patterns of lymphokine secretion lead to different functional properties," Annual Review of Immunology, vol. 7, pp. 145-173, 1989.

[55] T. A. Wynn, "Fibrotic disease and the $\mathrm{T}(\mathrm{H}) 1 / \mathrm{T}(\mathrm{H}) 2$ paradigm," Nature Reviews Immunology, vol. 4, no. 8, pp. 583594, 2004.

[56] F. Boin, U. De Fanis, S. J. Bartlett, F. M. Wigley, A. Rosen, and V. Casolaro, "T cell polarization identifies distinct clinical phenotypes in scleroderma lung disease," Arthritis and Rheumatism, vol. 58, no. 4, pp. 1165-1174, 2008.

[57] S. P. Atamas, V. V. Yurovsky, R. Wise et al., "Production of type 2, cytokines by CD8+ lung cells is associated with greater decline in pulmonary function in patients with systemic sclerosis," Arthritis and Rheumatism, vol. 42, no. 6, pp. 1168 1178, 1999.

[58] E. Scala, S. Pallotta, A. Frezzolini et al., "Cytokine and chemokine levels in systemic sclerosis: relationship with cutaneous and internal organ involvement," Clinical and Experimental Immunology, vol. 138, no. 3, pp. 540-546, 2004.

[59] D. M. Wuttge, M. Wildt, P. Geborek, F. A. Wollheim, A. Scheja, and A. Åkesson, "Serum IL-15 in patients with early systemic sclerosis: a potential novel marker of lung disease," Arthritis Research and Therapy, vol. 9, no. 5, article R85, 2007.

[60] I. Gutcher, M. K. Donkor, Q. Ma, A. Y. Rudensky, R. A. Flavell, and M. O. Li, "Autocrine transforming growth 
factor- $\beta 1$ promotes in vivo Th17 cell differentiation," Immunity, vol. 34, no. 3, pp. 396-408, 2011.

[61] K. Kurasawa, K. Hirose, H. Sano et al., "Increased interleukin-17 production in patients with systemic sclerosis," Arthritis and Rheumatism, vol. 43, no. 11, pp. 2455-2463, 2000.

[62] T. R. D. J. Radstake, L. van Bon, J. Broen et al., "The pronounced Th17 profile in systemic sclerosis (SSc) together with intracellular expression of TGF $\beta$ and IFN $\gamma$ distinguishes SSc phenotypes," PLoS ONE, vol. 4, no. 6, article e5903, 2009.

[63] M. Murata, M. Fujimoto, T. Matsushita et al., "Clinical association of serum interleukin-17 levels in systemic sclerosis: is systemic sclerosis a Th17 disease?" Journal of Dermatological Science, vol. 50, no. 3, pp. 240-242, 2008.

[64] P. Gourh, F. C. Arnett, S. Assassi et al., "Plasma cytokine profiles in systemic sclerosis: associations with autoantibody subsets and clinical manifestations," Arthritis Research and Therapy, vol. 11, no. 5, article R147, 2009.

[65] K. Komura, M. Fujimoto, M. Hasegawa et al., "Increased serum interleukin 23 in patients with systemic sclerosis," Journal of Rheumatology, vol. 35, no. 1, pp. 120-125, 2008.

[66] W. Ouyang, J. K. Kolls, and Y. Zheng, "The biological functions of T helper 17 cell effector cytokines in inflammation," Immunity, vol. 28, no. 4, pp. 454-467, 2008.

[67] S. Trifari, C. D. Kaplan, E. H. Tran, N. K. Crellin, and H. Spits, "Identification of a human helper $\mathrm{T}$ cell population that has abundant production of interleukin 22 and is distinct from TH-17, TH1 and TH2 cells," Nature Immunology, vol. 10, no. 8, pp. 864-871, 2009.

[68] M.-E. Truchetet, N. C. Brembilla, E. Montanari, Y. Allanore, and C. Chizzolini, "Increased frequency of circulating Th22 in addition to Th17 and Th2 lymphocytes in systemic sclerosis: association with interstitial lung disease," Arthritis Research \& Therapy, vol. 13, no. 5, article R166, 2011.

[69] R. Lafyatis, C. O'Hara, C. A. Feghali-Bostwick, and E. Matteson, "B cell infiltration in systemic sclerosis-associated interstitial lung disease," Arthritis and Rheumatism, vol. 56, no. 9, pp. 3167-3168, 2007.

[70] M. L. Whitfield, D. R. Finlay, J. I. Murray et al., "Systemic and cell type-specific gene expression patterns in scleroderma skin," Proceedings of the National Academy of Sciences of the United States of America, vol. 100, no. 21, pp. 12319-12324, 2003.

[71] S. Sato, M. Fujimoto, M. Hasegawa, K. Takehara, and T. F. Tedder, "Altered B lymphocyte function induces systemic autoimmunity in systemic sclerosis," Molecular Immunology, vol. 41, no. 12, pp. 1123-1133, 2004.

[72] S. Sato, M. Hasegawa, M. Fujimoto, T. F. Tedder, and K. Takehara, "Quantitative genetic variation in CD19 expression correlates with autoimmunity," Journal of Immunology, vol. 165, no. 11, pp. 6635-6643, 2000.

[73] D. Daoussis, S. N. C. Liossis, A. C. Tsamandas et al., "Experience with rituximab in scleroderma: results from a 1year, proof-of-principle study," Rheumatology, vol. 49, no. 2, pp. 271-280, 2009.

[74] F. O. Martinez, A. Sica, A. Mantovani, and M. Locati, "Macrophage activation and polarization," Frontiers in Bioscience, vol. 13, no. 2, pp. 453-461, 2008.

[75] N. Higashi-Kuwata, M. Jinnin, T. Makino et al., "Characterization of monocyte/macrophage subsets in the skin and peripheral blood derived from patients with systemic sclerosis," Arthritis Research and Therapy, vol. 12, no. 4, article R128, 2010.

[76] S. K. Mathai, M. Gulati, X. Peng et al., "Circulating monocytes from systemic sclerosis patients with interstitial lung disease show an enhanced profibrotic phenotype," Laboratory Investigation, vol. 90, no. 6, pp. 812-823, 2010.

[77] F. Zuo, N. Kaminski, E. Eugui et al., "Gene expression analysis reveals matrilysin as a key regulator of pulmonary fibrosis in mice and humans," Proceedings of the National Academy of Sciences of the United States of America, vol. 99, no. 9, pp. 6292-6297, 2002.

[78] R. C. A. Dancer, A. M. Wood, and D. R. Thickett, "Metalloproteinases in idiopathic pulmonary fibrosis," European Respiratory Journal, vol. 38, no. 6, pp. 1461-1467, 2011.

[79] I. O. Rosas, T. J. Richards, K. Konishi et al., "MMP1 and MMP7 as potential peripheral blood biomarkers in idiopathic pulmonary fibrosis," PLoS Medicine, vol. 5, no. 4, article e93, pp. 623-633, 2008.

[80] P. Moinzadeh, T. Krieg, M. Hellmich et al., "Elevated MMP7 levels in patients with systemic sclerosis: correlation with pulmonary involvement," Experimental Dermatology, vol. 20, no. 9, pp. 770-773, 2011.

[81] W. U. Kim, S. Y. Min, M. L. Cho et al., "Elevated matrix metalloproteinase-9 in patients with systemic sclerosis," Arthritis Research \& Therapy, vol. 7, no. 1, pp. R71-R79, 2005.

[82] G. N. Andersen, K. Nilsson, J. Pourazar et al., "Bronchoalveolar matrix metalloproteinase 9 relates to restrictive lung function impairment in systemic sclerosis," Respiratory Medicine, vol. 101, no. 10, pp. 2199-2206, 2007.

[83] M. Manetti, S. Guiducci, E. Romano et al., "Increased serum levels and tissue expression of matrix metalloproteinase- 12 in patients with systemic sclerosis: correlation with severity of skin and pulmonary fibrosis and vascular damage," Annals of the Rheumatic Diseases, vol. 71, no. 6, pp. 1064-1072, 2012.

[84] K. Kikuchi, M. Kubo, S. Sato, M. Fujimoto, and K. Tamaki, "Serum tissue inhibitor of metalloproteinases in patients with systemic sclerosis," Journal of the American Academy of Dermatology, vol. 33, no. 6, pp. 973-978, 1995.

[85] F. Chua and G. J. Laurent, "Neutrophil elastase: mediator of extracellular matrix destruction and accumulation," Proceedings of the American Thoracic Society, vol. 3, no. 5, pp. 424427, 2006.

[86] T. Hara, F. Ogawa, K. Yanaba et al., "Elevated serum concentrations of polymorphonuclear neutrophilic leukocyte elastase in systemic sclerosis: association with pulmonary fibrosis," Journal of Rheumatology, vol. 36, no. 1, pp. 99-105, 2009.

[87] M. Dziadzio, W. Usinger, A. Leask et al., "N-terminal connective tissue growth factor is a marker of the fibrotic phenotype in scleroderma," QJM-Monthly Journal of the Association of Physicians, vol. 98, no. 7, pp. 485-492, 2005.

[88] S. Sato, T. Nagaoka, M. Hasegawa et al., "Serum levels of connective tissue growth factor are elevated in patients with systemic sclerosis: association with extent of skin sclerosis and severity of pulmonary fibrosis," Journal of Rheumatology, vol. 27, no. 1, pp. 149-154, 2000.

[89] M. Dziadzio, R. E. Smith, D. J. Abraham, C. M. Black, and C. P. Denton, "Circulating levels of active transforming growth factor $\beta 1$ are reduced in diffuse cutaneous systemic sclerosis and correlate inversely with the modified Rodnan skin score," Rheumatology, vol. 44, no. 12, pp. 1518-1524, 2005.

[90] F. Ogawa, K. Shimizu, E. Muroi, T. Hara, and S. Sato, "Increasing levels of serum antioxidant status, total antioxidant power, in systemic sclerosis," Clinical Rheumatology, vol. 30, no. 7, pp. 921-925, 2011.

[91] F. Ogawa, K. Shimizu, E. Muroi et al., "Serum levels of 8 -isoprostane, a marker of oxidative stress, are elevated in 
patients with systemic sclerosis," Rheumatology, vol. 45, no. 7, pp. 815-818, 2006.

[92] L. Scussel-Lonzetti, F. Joyal, J. P. Raynauld et al., "Predicting mortality in systemic sclerosis: analysis of a cohort of 309 French Canadian patients with emphasis on features at diagnosis as predictive factors for survival," Medicine, vol. 81, no. 2, pp. 154-167, 2002.

[93] C. Ferri, G. Valentini, F. Cozzi et al., "Systemic sclerosis: demographic, clinical, and serologic features and survival in 1,012 Italian patients," Medicine, vol. 81, no. 2, pp. 139-153, 2002.

[94] C. Muangchan, S. Harding, S. Khimdas et al., "C-reactive protein (CRP) is associated with highdisease activity in systemic sclerosis: results from the Canadian Scleroderma Research Group (CSRG)," Arthritis Care \& Research (Hoboken). In press.

[95] T. Ohtsuka, "Serum interleukin-6 level is reflected in elevated high-sensitivity C-reactive protein level in patients with systemic sclerosis," Journal of Dermatology, vol. 37, no. 9, pp. 801-806, 2010.

[96] G. W. Lee, T. H. Lee, and J. T. Vilcek, "TSG-14, a tumor necrosis factor- and IL-1-inducible protein, is a novel member of the pentaxin family of acute phase proteins," Journal of Immunology, vol. 150, no. 5, pp. 1804-1812, 1993.

[97] Y. Iwata, A. Yoshizaki, F. Ogawa et al., "Increased serum pentraxin 3 in patients with systemic sclerosis," Journal of Rheumatology, vol. 36, no. 5, pp. 976-983, 2009.

[98] S. Nagpal, S. Na, and R. Rathnachalam, "Noncalcemic actions of vitamin D receptor ligands," Endocrine Reviews, vol. 26, no. 5, pp. 662-687, 2005.

[99] P. Caramaschi, A. Dalla Gassa, O. Ruzzenente et al., "Very low levels of vitamin D in systemic sclerosis patients," Clinical Rheumatology, vol. 29, no. 12, pp. 1419-1425, 2010.

[100] Y. Arnson, H. Amital, N. Agmon-Levin et al., "Serum 25$\mathrm{OH}$ vitamin D concentrations are linked with various clinical aspects in patients with systemic sclerosis: a retrospective cohort study and review of the literature," Autoimmunity Reviews, vol. 10, no. 8, pp. 490-494, 2011.

[101] A. Vacca, C. Cormier, M. Piras, A. Mathieu, A. Kahan, and Y. Allanore, "Vitamin D deficiency and insufficiency in 2 independent cohorts of patients with systemic sclerosis," Journal of Rheumatology, vol. 36, no. 9, pp. 1924-1929, 2009.

[102] C. Fonseca, D. Abraham, and E. A. Renzoni, "Endothelin in pulmonary fibrosis," American Journal of Respiratory Cell and Molecular Biology, vol. 44, no. 1, pp. 1-10, 2010.

[103] A. D. Cambrey, N. K. Harrison, K. E. Dawes et al., "Increased levels of endothelin-1 in bronchoalveolar lavage fluid from patients with systemic sclerosis contribute to fibroblast mitogenic activity in vitro," American Journal of Respiratory Cell and Molecular Biology, vol. 11, no. 4, pp. 439-445, 1994.

[104] D. J. Abraham, R. Vancheeswaran, M. R. Dashwood et al., "Increased levels of endothelin-1 and differential endothelin type A and B receptor expression in scleroderma-associated fibrotic lung disease," American Journal of Pathology, vol. 151, no. 3, pp. 831-841, 1997.

[105] H. Ihn, S. Sato, M. Fujimoto, K. Takehara, and K. Tamaki, "Increased serum levels of soluble vascular cell adhesion molecule-1 and E-selectin in patients with systemic sclerosis," British Journal of Rheumatology, vol. 37, no. 11, pp. 11881192, 1998.

[106] A. Kuryliszyn-Moskal, P. A. Klimiuk, and S. Sierakowski, "Soluble adhesion molecules (sVCAM-1, sE-selectin), vascular endothelial growth factor (VEGF) and endothelin1 in patients with systemic sclerosis: relationship to organ systemic involvement," Clinical Rheumatology, vol. 24, no. 2, pp. 111-116, 2005.

[107] R. Vancheeswaran, T. Magoulas, G. Efrat et al., "Circulating endothelin-1 levels in systemic sclerosis subsets-a marker of fibrosis or vasular dysfunction?" Journal of Rheumatology, vol. 21, no. 10, pp. 1838-1844, 1994.

[108] C. Mihai and J. W. C. Tervaert, "Anti-endothelial cell antibodies in systemic sclerosis," Annals of the Rheumatic Diseases, vol. 69, no. 2, pp. 319-324, 2010.

[109] H. Ihn, S. Sato, M. Fujimoto et al., "Characterization of autoantibodies to endothelial cells in systemic sclerosis (SSc): association with pulmonary fibrosis," Clinical and Experimental Immunology, vol. 119, no. 1, pp. 203-209, 2000.

[110] D. Launay, M. Humbert, A. Berezne et al., "Clinical characteristics and survival in systemic sclerosis-related pulmonary hypertension associated with interstitial lung disease," Chest, vol. 140, no. 4, pp. 1016-1024, 2011.

[111] B. E. Schreiber, C. J. Valerio, G. J. Keir et al., "Improving the detection of pulmonary hypertension in systemic sclerosis using pulmonary function tests," Arthritis and Rheumatism, vol. 63, no. 11, pp. 3531-3539, 2011.

[112] T. J. Corte, S. J. Wort, P. S. MacDonald et al., "Pulmonary function vascular index predicts prognosis in idiopathic interstitial pneumonia," Respirology, vol. 17, no. 4, pp. 674680, 2012.

[113] S. M. Arcasoy, J. D. Christie, V. A. Ferrari et al., "Echocardiographic assessment of pulmonary hypertension in patients with advanced lung disease," American Journal of Respiratory and Critical Care Medicine, vol. 167, no. 5, pp. 735-740, 2003.

[114] N. Nagaya, T. Nishikimi, Y. Okano et al., "Plasma brain natriuretic peptide levels increase in proportion to the extent of right ventricular dysfunction in pulmonary hypertension," Journal of the American College of Cardiology, vol. 31, no. 1, pp. 202-208, 1998.

[115] C. T. Gan, G. P. McCann, J. T. Marcus et al., "NTproBNP reflects right ventricular structure and function in pulmonary hypertension," European Respiratory Journal, vol. 28, no. 6, pp. 1190-1194, 2006.

[116] H. H. Leuchte, R. A. Baumgartner, M. El Nounou et al., "Brain natriuretic peptide is a prognostic parameter in chronic lung disease," American Journal of Respiratory and Critical Care Medicine, vol. 173, no. 7, pp. 744-750, 2006.

[117] T. J. Corte, S. J. Wort, M. A. Gatzoulis et al., "Elevated brain natriuretic peptide predicts mortality in interstitial lung disease," European Respiratory Journal, vol. 36, no. 4, pp. 819-825, 2010. 


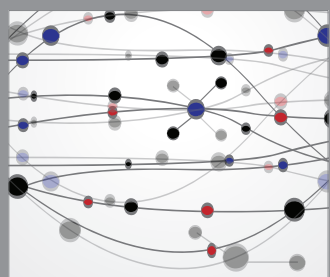

The Scientific World Journal
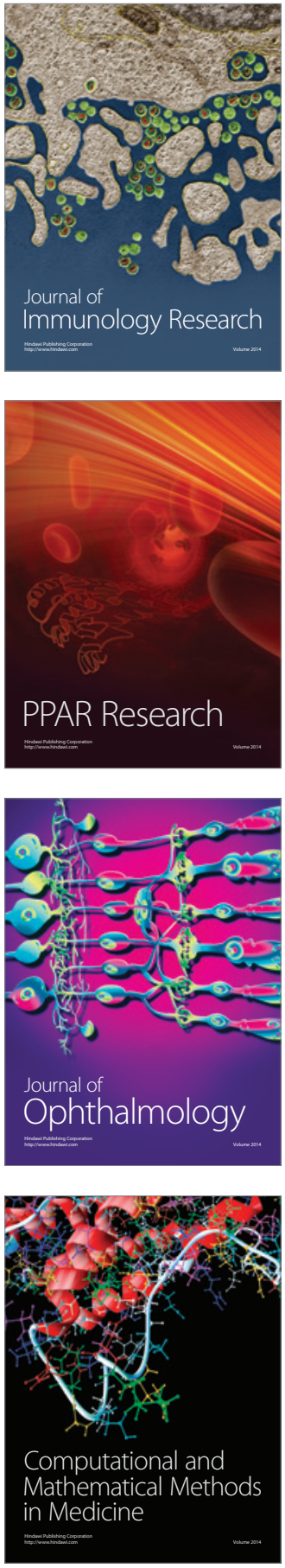

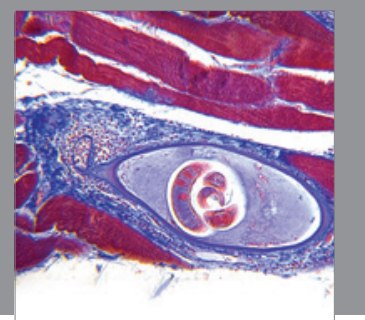

Gastroenterology

Research and Practice
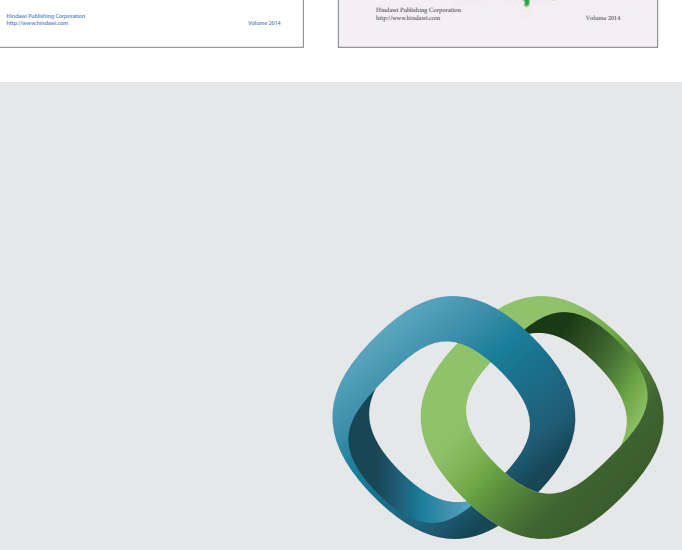

\section{Hindawi}

Submit your manuscripts at

http://www.hindawi.com
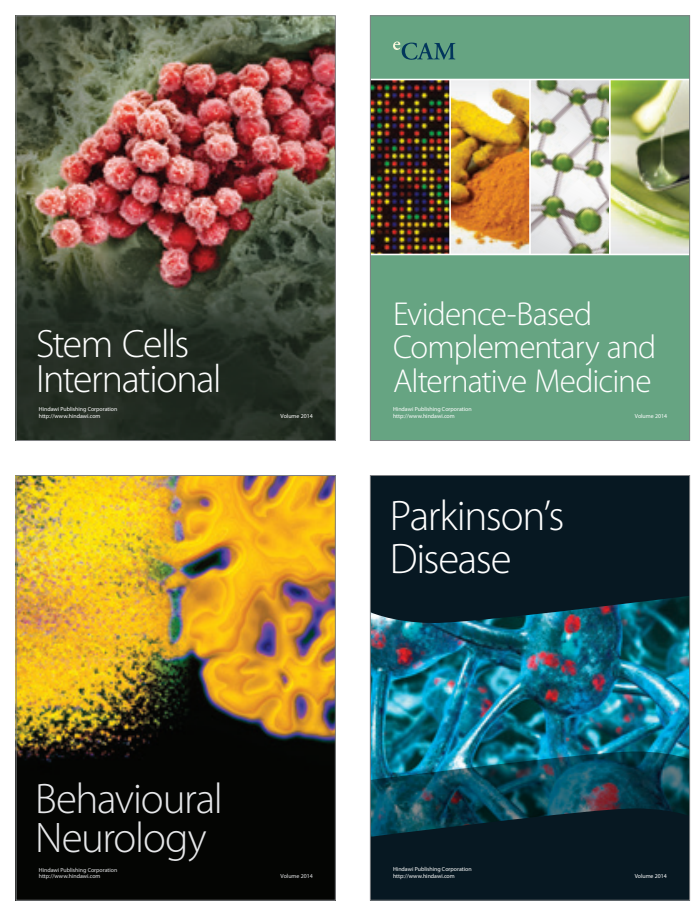

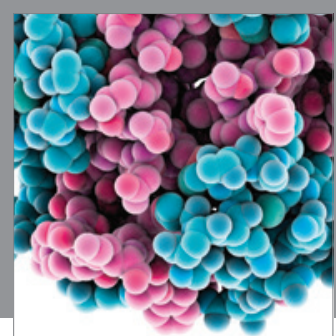

Journal of
Diabetes Research

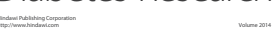

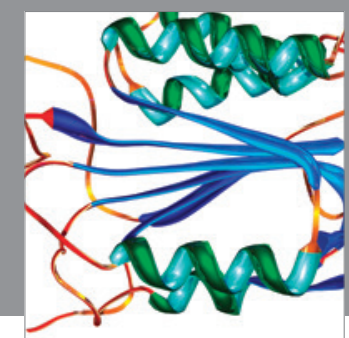

Disease Markers
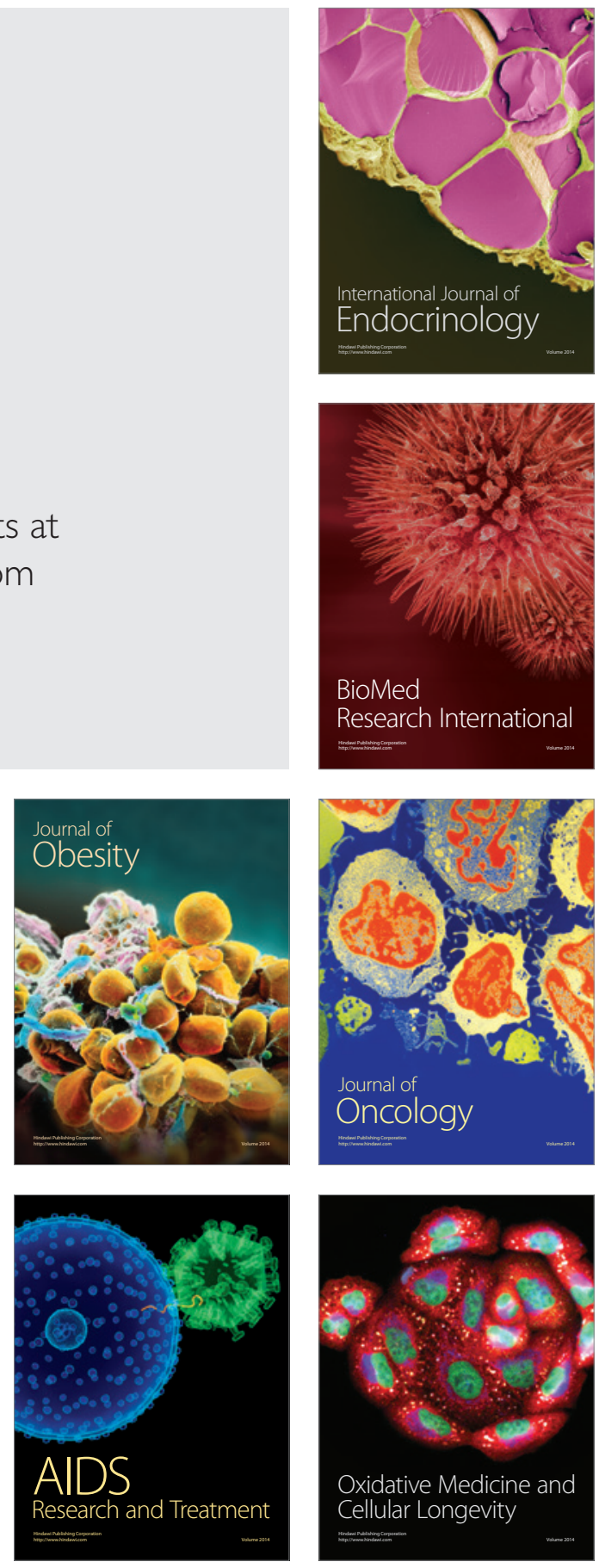